\title{
Developing a Novel Trust Model for the Best Resource Selection among the Resources in Grid Environment
}

\author{
Sourav Gayen \\ M-Tech (CST), Dept of \\ C.S.E/IT \\ Dr. B.C Roy Engineering \\ College, Durgapur
}

\author{
Avijit Bhowmick \\ Asst.Professor, Dept of \\ C.S.E/IT \\ Dr. B.C Roy Engineering \\ College, Durgapur
}

\author{
Biswajit Upadhyay \\ Asst. Professor, Dept of \\ C.S.E/IT \\ Aryabhatta Institute of \\ Engineering and Management, \\ Durgapur
}

\begin{abstract}
Grid computing is a wide area distributed parallel computing environment where it focuses on communication among different heterogeneous devices and harnesses unused processor cycles of all computers in a network for solving critical problems too intensive for any stand-alone machine. In this scenario security is vital concern because of dynamic allocation of users and resources. Among all other security issues trust establishment is given first priority.In this paper, we propose a novel model for resource selection in Grid computing environment. This model is truly unique model where decision is based on computed total trust which includes transaction, feedbacks, reputation, similarity, activity, popularity and obviously the total trust of the resources. The final decision is depend on the total trust of each resource. So the main motto of our project is to retrieve the best resource among the resources in grid environment. This paper provides a model which will allow only dependable transactions in grid by using total trust as a measure for the resources.
\end{abstract}

\section{Keywords}

Grid Computing, Trust, Reputation

\section{INTRODUCTION}

Grid computing is focused on the ability to support computation across multiple administrative domains that sets it apart from traditional distributed computing. Grids offer a way of using the information technology resources optimally inside an organization involving virtualization of computing resources. Its concept of support for multiple administrative policies and security authentication and authorization mechanisms enables it to be distributed over a local, metropolitan, or wide-area network. Grid computing provides huge processing power in a distributed environment with variety of resources. The resources in Grid are shared in a flexible, coordinated and secured manner. Most of the Grid applications involve very large data bases with highly secured data. [1]A well-known example of grid computing in the public domain is the ongoing SETI (Search for Extraterrestrial Intelligence) @ Home project in which thousands of people are sharing the unused processor cycles of their PCs in the vast search for signs of "rational" signals from outer space. According to John Patrick, IBM's vicepresident for Internet strategies, "the next big thing will be grid computing". Grid computing requires the use of software that can divide and farm out pieces of a program to as many as several thousand computers. Grid computing appears to be a promising trend for three reasons: (1) its ability to make more cost-effective use of a given amount of computer resources, (2) as a way to solve problems that can't be approached without an enormous amount of computing power, and (3) because it suggests that the resources of many computers can be cooperatively and perhaps synergistically harnessed and managed as a collaboration toward a common objective. In some grid computing systems, the computers may collaborate rather than bein directed by one managing computer.

\section{Definition of Reputation:}

Reputation is a summary of one's previous or past experiences. a specific community, presented in a manner that can help other community members to make decisions. According to Farag Azzedin and Muthucumaru Maheswaran, "The reputation of an entity is an expectation of its behavior based on other entities' observations or information about the entity's past behavior within a specific context at a given time." So Reputation refers to the value we attribute to a specific Resource, including agents, services, and persons in the Grid, based on the trust revealed by it in the past. It reflects the perception that one has of another's intentions and norms. Resource reputation provides a way of assigning quality or value in regards to a resource. If a resource is known to provide certain qualities over a period of time irrespective of its restrictions, then it is assumed to have good reputation.

\section{Definition Of Trust:}

Trust plays a role across many disciplines which attempted to define the concept. The definition of trust that we will use in this paper is as follows:

"Trust is the firm belief in the competence of an entity to act as expected such that this firm beliefis not a fixed value associated with the entity but rather it is subject to the entity's behavior and applies only within a specific context at a given time." According to Gambetta [2]: "When we say we trust someone or that someone is trustworthy, we implicitly mean that the probability that he will perform an action that is beneficial or at least not detrimental to us is high enough for us to consider engaging in some form of cooperation with him. Correspondingly, when we say that someone is untrustworthy, we imply that that probability is low enough for us to refrain from doing so". 


\section{Related Works:}

A reputation-based structure is offered by Li xiong and liu [5]. They state that feed back values only are not adequate for the computation of trust and reputation. Y. Wang and J. Vassileva [6] recommend a reputation model based on Bayesian network. According to their model the peers requests are poles apart in different situations. Selcuk et al. suggests in [7] a reputation based trust management system in which the dependability is calculated based on prior transactions. Ayman Tajeddine et al. in [8] propose a very impressive reputation based trust model. In this approach the initiator host calculates reputation value of target host based on its previous experiences and grouped feedbacks from other resources. F.Azzedin,M.Maheswaran [9] argue about managing trust in grid by proposing a performance trust management model. Trust levels are graded from a to f. Both direct and indirect trust are considered. Gui Xiaolin, Xie Bing [10] propose a trust model based on behavior tracks. Attenuation function is corporated for decaying factor. Baolin $\mathrm{Ma}$ et al in[11] present a reputation based trusted model .Their model considers both direct feed back and feedback from other entities Direct trust is given with more weightage than the indirect score.Beulah kurian, Gregor von laszewki [12] provide a way for efficient resource choice by making an allowance for Eigen trust algorithm. Their approach is related to Azzedin approach [9] except for a new parameter framework.

\section{Proposed Model:}

Our proposed model represent a new model after survey the related models[[8] and [13].In the model [13], P.Srivaramangai and R.Srinivasan uses rank correlation method for removing biased feed backs. It uses both direct trust and indirect trust. Direct trust is calculated from the transaction Table which are done directly by the resources and is given higher weightage. Indirect trust is measured by getting feed backs from entities in the same domain and also from other domains. In the model [8], Ayman Tajeddine, Ayman Kayssi, Ali Chehab and Hassan Artail represent a new comprehensive model for the calculation of reputation values and the determination of trust decisions. They shows the differentiate between two types of trust that affect the final decision of a host of whether to interact with another target host or not.Our model uses Kendall's tau(T) rank correlation to removed the needless feedbacks of each resource.Here we calculate the direct trusts(in same and other domain) and calculate the total indirect trusts for same and other domain by considering different parameters such as similarity, activity and popularity.Actually our model is basically to easily select the best resource from the grid environment according to their total trust. In this model, at first we calculate the feedback of each resource with respect to the other resources from the transaction table(which is given by us) and then calculate the reputation of each resource given by the other resources(may be in the same or other domain). After that we calculate DT and TIT(ITSd+ITOd) with parameters similarity,activity and popularity.And finally we calculate TT(Total Trust).Now According the maximum of TT we can easily clear to pick the best resource from the network. Indirect Trust calculation is totally depends upon the following parameters:

\subsection{Activity:}

The activity of a host depends on its total number of interactions with other Recommenders. This trait reflects the activity level of a host and how much its information is reliable and up-to-date.

\author{
Activity \\ $=\frac{\text { Total no. of interaction by Recommenders }}{\text { Total no. of interaction by all Recommenders }}$
}

\subsection{Similarity:}

The similarity of a host with another host is based on the similarity of their reputation values. The closer their values, the closer their evaluation procedures, and thus the more one's recommendation will be credible to the other. $P$. Srivaramangai \& Dr. R. Srinivasan uses Spearman's rho method to calculate similarity in their model[13].But here we calculate the similarity through the Kendall's tau(T) rank correlation formula because for the following reasons[4]..

- In practice, Spearman's rho is larger than Kendall's tau(T) rank correlation.

- Kendall's tau(T) rank correlation has more attractive quality over Spearman's rho.

- It has intuitive interpretation.

$$
\text { Similarity }=\frac{\sum C-\sum D}{\sum C+\sum D}
$$

\section{OR}

$$
\begin{aligned}
& \text { Similarity }=\frac{S}{\frac{1}{2} n(n-1)} \\
& \text { Where C=Concordant. } \\
& \text { D=Discordant. } \\
& \mathrm{S}=\sum \mathbf{C}-\sum \mathbf{D}
\end{aligned}
$$

And $n=$ Total No of Entities or Resources.

\subsection{Popularity:}

A host's popularity is based on the number of interactions hosts have had with this specific host. A host's popularity determines the degree to which this host is well-liked and accepted among other hosts.

$$
\begin{aligned}
& \text { popularity } \\
& =\frac{\text { Total no. of interaction with initiator }}{\text { Total no. of interaction by all other hosts }}
\end{aligned}
$$

\section{SYMBOLS USED IN ALGORITHM}

Trsn_ $R_{i}=$ Transaction of The Resource $R_{i}$ $\operatorname{FbSd}\left(\frac{R_{j}}{R_{i}}\right)=$

Feed Back of $R_{j}$ given by $R_{i}$ in Same DomainFbOd $\left(\frac{R_{j}}{R_{i}}\right)=$ Feed Back of $R_{j}$ given by $R_{i}$ in Other DomainRep $\left(\frac{R_{j}}{R_{i}}\right)=$ Reputation of $R_{j}$ given by $R_{i}$. DT $\left(\frac{R j}{R i}\right)=$ DirectTrust of $R_{j}$ by $R_{i}$. ITSd_ $R_{i}=$ IndirectTrust of $R_{i}$ in Same DomainITOd_R $R_{i}$ $=$ IndirectTrust of $R_{i}$ in Other DomainTIT $R_{R_{i}}$

$=$ Total Indirect Trust of $R_{i}$ Sim_ $R_{i}$

= Similaritry of $R_{i}$ Acty_ $R_{i}=$ Activity of $R_{i}$ Popty_ $R_{i}=$ Popularity of $R_{i}$ $T T_{-} R_{i}=$ Total Trust of $R_{i}$ 


\section{Algorithm:}

Step-1: At first create a Transaction Table by the given formula.

Trsn_ $R_{1}=\frac{\text { Previously total no of successfull interaction within a time period } T}{\text { Previously total no of interaction within a time period } T}$

Step-2: Calculate feed-backs of each resource (same and different domain)from the Transaction table and store into the Feed-back Table.

Step-3: Calculate Reputation of each resource from the Feed-Back Table and store into the Reputation Table by the formula..

$$
\operatorname{Rep}\left(\frac{R_{j}}{R_{i}}\right)=\text { Reputation of } R_{j} \text { given by } R_{i}
$$

where, $\boldsymbol{R e p}\left(\mathbf{R}_{\mathbf{j}} / \mathbf{R}_{\boldsymbol{i}}\right)=$ Direct feedback by $\mathbf{R}_{\mathrm{i}}+$ (sum of total feedbacks of $\mathrm{R}_{\mathrm{j}}$ in the same domain)*a+( sum of total feedbacks of $\mathrm{R}_{\mathrm{j}}$ in the other domain) $* \mathrm{~b}$. where $a=0.5$ and $b=0.25$ are the threshold values of the weight for same domain and the other domain respectively and $(\mathrm{a}>\mathrm{b})$.

Step-4: Calculate the Direct Trust(DT) of each resource by $\mathbf{D T}\left(\frac{\mathbf{R j}}{\mathbf{R i}}\right)$ where,

$$
D T\left(\frac{R j}{R i}\right)=\frac{\text { Feedbak of } R_{j} \text { by } R_{i}+\text { Reputation by } R_{i}}{2}
$$

Step-5: Calculate Indirect Trusts(ITSd and ITOd) for same domain and also for other domain with the parameters...

I. Similarity [Using Kendall's tau(T) rank correlation Formula]

II. Calculate Activity (Acty) of each resource.

III. Calculate Popularity(Popty) of each resource

Indirect Trust $($ IT $)=$ Sim + Acty + Popty .

Step-6: Calculate Total Trust(TT) where,

TT=DT+ITSd+ITOd.

Step-7: Calculate the $\max (\mathrm{TT})$.

Step-8: Check $\max (\mathrm{TT})>1$ if NO then we get the resource and stop .Otherwise continue.

Step-9: Check $\max (\mathrm{TT})>1$ if NO then we get the resource and stop .Otherwise continue.

Step-10: Calculate the $\max (\mathrm{DT})$ among the selected resources.

Step-11: Check $\max (\mathrm{DT})>1$ if NO then we get the resource and stop. Otherwise continue..

Step-12: Calculate the Total Indirect Trust(IT_1+IT_2) for selected resources and calculate the maximum of Total Indirect Trust(TIT).

Step-13: Check $\max (\mathrm{TIT})>1$, if No then we get the final resource and stop, otherwise continue from Step-1 with selected resource.

Step-14:Get The Selected Resource.

\section{Calculation of Reputation:}

Here, we calculate the reputation of each resource given by the another resource from the reputation table. For example, calculation of the reputation of a resource $R_{9}$ given by the resources $R_{1}, R_{2}, R_{3}, R_{4}, R_{5}, R_{6}, R_{7}, R_{8}, R_{10}, R_{11}, R_{12}, R_{13}, R_{14}$ $\mathrm{R}_{15}$ are as follows..

$\operatorname{Rep}\left(\frac{R_{9}}{R_{1}}\right)=$ Direct Feed-back from $\mathrm{R}_{1}+($ Sum of Feed-backs given by the resources in the same domain $) * 0.5++($ Sum of Feed-backs given by the resources from the other domain) $* 0.2$.

$=0.667+(0.875+0.733+0.889+0.556+1+0.99+0.204) * 0.5+(1+$ $0.7+0.525+0.909+0.875+0.733) * 0.25$.

$=0.667+2.6235+1.1855$

$=4.476$.

Similarly,

$\operatorname{Rep}\left(\frac{R_{9}}{R_{2}}\right)=0.154+1.926+0.9865=3.0665$.

$\operatorname{Rep}\left(\frac{R_{9}}{R_{3}}\right)=0.478+1.976+0.93825=3.39225$.

$\operatorname{Rep}\left(\frac{R_{9}}{R_{4}}\right)=0.556+1.5585+0.728=2.1145$.

$\operatorname{Rep}\left(\frac{R_{9}}{R_{5}}\right)=0.982+1.7355+0.72575=3.44325$.

$\operatorname{Rep}\left(\frac{R_{9}}{R_{6}}\right)=0.838+2.4055+0.97025=4.21375$.

$\operatorname{Rep}\left(\frac{R_{9}}{R_{7}}\right)=0.5+2.0505+0.9505=3.501$.

$\operatorname{Rep}\left(\frac{R_{9}}{R_{8}}\right)=0.125+1.7415+0.8465=2.713$

$\operatorname{Rep}\left(\frac{R_{9}}{R_{10}}\right)=0.579+0.825+1.464=2.868$.

$\operatorname{Rep}\left(\frac{R_{9}}{R_{11}}\right)=0.957+1.3595+0.9695=3.286$.

$\operatorname{Rep}\left(\frac{R_{9}}{R_{12}}\right)=0.957+1.36025+0.9695=3.28675$.

$\operatorname{Rep}\left(\frac{R_{9}}{R_{13}}\right)=0.9+1 \cdot 28925+1 \cdot 526=3 \cdot 71525$.

$\operatorname{Rep}\left(\frac{R_{9}}{R_{14}}\right)=0.2+1.0915+1.4725=2.764$.

$\operatorname{Rep}\left(\frac{R_{9}}{R_{15}}\right)=0.407+1.49625+1.3175=3.22075$ 


\section{Flow-Chart of the Trust Model}

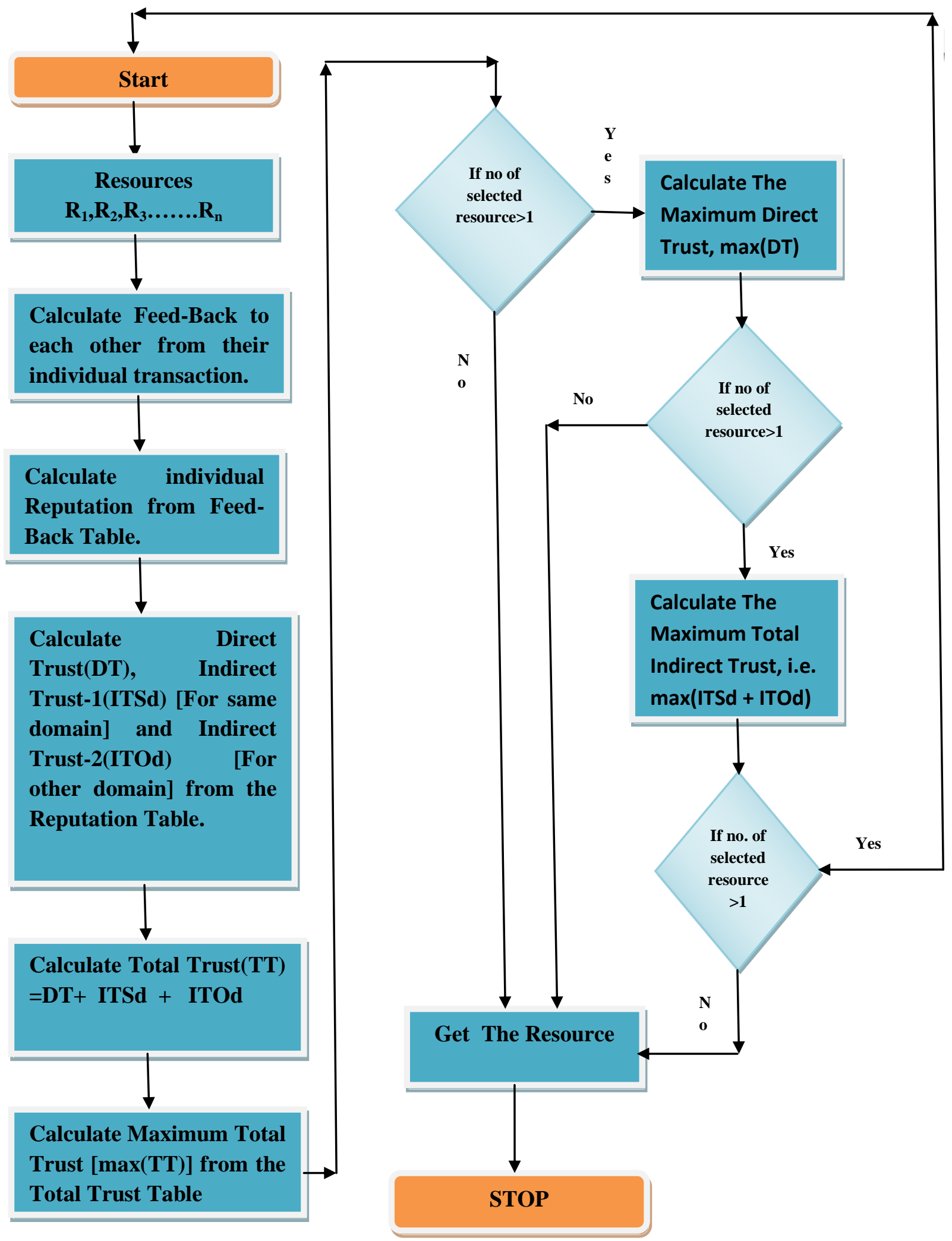




\begin{tabular}{|c|c|c|c|c|c|c|c|c|c|c|c|c|c|c|c|}
\hline \multicolumn{16}{|c|}{ REPUTATION TABLE } \\
\hline & $\overline{\text { R1 }}$ & $\overline{\mathbf{R} 2}$ & $\mathbf{R 3}$ & $\overline{\mathbf{R} 4}$ & $\overline{R 5}$ & R6 & R7 & R8 & R9 & R10 & R11 & R12 & R13 & $\begin{array}{l}\text { R14 } \\
\end{array}$ & $\mathbf{R 1 5}$ \\
\hline R1 & & 3.301 & 3.478 & $\begin{array}{l}2.57 \\
4\end{array}$ & $\begin{array}{l}3.07 \\
6\end{array}$ & 3.71 & $\begin{array}{l}3.18 \\
6\end{array}$ & $\begin{array}{l}2.83 \\
8\end{array}$ & $\begin{array}{l}3.70 \\
8\end{array}$ & $\begin{array}{l}3.72 \\
2\end{array}$ & $\begin{array}{l}3.30 \\
8\end{array}$ & 4.228 & $\begin{array}{l}3.55 \\
4\end{array}$ & 2.94 & $\mathbf{3 . 3 3}$ \\
\hline $\mathbf{R} 2$ & $\begin{array}{l}4.41 \\
3\end{array}$ & & 3.215 & $\begin{array}{l}2.65 \\
9\end{array}$ & $\begin{array}{l}2.83 \\
9\end{array}$ & $\begin{array}{l}4.06 \\
3\end{array}$ & $\begin{array}{l}3.40 \\
4\end{array}$ & $\begin{array}{l}2.87 \\
3\end{array}$ & $\begin{array}{l}3.47 \\
6\end{array}$ & $\begin{array}{l}3.51 \\
5\end{array}$ & 3.37 & 3.785 & $\begin{array}{l}3.89 \\
\end{array}$ & 2.964 & $\begin{array}{l}3.66 \\
4\end{array}$ \\
\hline R3 & \begin{tabular}{|l|}
4.34 \\
2 \\
\end{tabular} & 3.326 & & $\begin{array}{l}2.45 \\
1\end{array}$ & $\begin{array}{l}2.98 \\
2\end{array}$ & $\begin{array}{l}3.9 \\
\end{array}$ & $\begin{array}{l}.47 \\
1 \\
\end{array}$ & $\begin{array}{l}2.88 \\
5 \\
\end{array}$ & $\begin{array}{l}3.51 \\
5 \\
\end{array}$ & $\begin{array}{l}3.06 \\
7 \\
\end{array}$ & $\begin{array}{l}3.32 \\
4 \\
\end{array}$ & 4.1 & $\begin{array}{l}3.92 \\
2 \\
\end{array}$ & 3.152 & 3.76 \\
\hline R4 & \begin{tabular}{|l|}
4.42 \\
\end{tabular} & \begin{tabular}{|l|l|}
3.22 \\
\end{tabular} & $\begin{array}{l}3.180 \\
5\end{array}$ & & $\begin{array}{l}2.95 \\
8\end{array}$ & $\begin{array}{l}3.97 \\
2\end{array}$ & $\begin{array}{l}3.42 \\
9\end{array}$ & $\begin{array}{l}2.89 \\
8\end{array}$ & $\begin{array}{l}3.36 \\
5\end{array}$ & $\begin{array}{l}3.17 \\
3\end{array}$ & $\begin{array}{l}3.00 \\
8\end{array}$ & 4.247 & $\begin{array}{l}4.01 \\
5\end{array}$ & 2.864 & $\begin{array}{l}3.71 \\
7\end{array}$ \\
\hline R5 & \begin{tabular}{|l|}
4.25 \\
4
\end{tabular} & 3.336 & 3.51 & $\begin{array}{l}2.82 \\
8\end{array}$ & & $\begin{array}{l}4.07 \\
5\end{array}$ & $\begin{array}{l}3.52 \\
6\end{array}$ & $\begin{array}{l}3.11 \\
3\end{array}$ & $\begin{array}{l}\mathbf{3 . 3 3} \\
\mathbf{3}\end{array}$ & $\begin{array}{l}3.21 \\
2\end{array}$ & 3.51 & 4.299 & $\begin{array}{l}3.41 \\
8\end{array}$ & 3.146 & $\begin{array}{l}3.47 \\
9\end{array}$ \\
\hline R6 & $\begin{array}{l}4.47 \\
6\end{array}$ & 3.098 & 3.188 & $\begin{array}{l}2.67 \\
6\end{array}$ & $\begin{array}{l}3.01 \\
9\end{array}$ & & 3.56 & $\begin{array}{l}2.67 \\
3\end{array}$ & $\begin{array}{l}3.21 \\
5\end{array}$ & 3.01 & $\begin{array}{l}3.54 \\
2\end{array}$ & 4.038 & 3.75 & 2.771 & 3.23 \\
\hline R7 & 4.47 & 3.251 & 3.25 & $\begin{array}{l}2.71 \\
3\end{array}$ & $\begin{array}{l}\begin{array}{l}3.07 \\
6\end{array} \\
\end{array}$ & $\begin{array}{l}3.91 \\
9\end{array}$ & & $\begin{array}{l}.82 \\
3\end{array}$ & $\begin{array}{l}3.46 \\
8\end{array}$ & $\begin{array}{l}2.91 \\
2\end{array}$ & 3.09 & 4.307 & $\begin{array}{l}3.54 \\
4\end{array}$ & 3.368 & $\begin{array}{l}3.63 \\
5\end{array}$ \\
\hline $\mathbf{R 8}$ & $\begin{array}{l}4.07 \\
8\end{array}$ & 3.161 & \begin{tabular}{|c|}
3.391 \\
\end{tabular} & $\begin{array}{l}2.63 \\
6\end{array}$ & 2.82 & $\begin{array}{l}3.86 \\
3\end{array}$ & $\begin{array}{l}3.35 \\
7\end{array}$ & & $\begin{array}{l}3.49 \\
3\end{array}$ & $\begin{array}{l}3.38 \\
4\end{array}$ & $\begin{array}{l}3.39 \\
1\end{array}$ & 3.401 & $\begin{array}{l}3.49 \\
4\end{array}$ & 3.382 & $\begin{array}{l}3.76 \\
7\end{array}$ \\
\hline R9 & $\begin{array}{l}4.47 \\
6\end{array}$ & 3.067 & 3.392 & $\begin{array}{l}2.11 \\
5\end{array}$ & $\begin{array}{l}3.44 \\
3\end{array}$ & $\begin{array}{l}4.21 \\
4\end{array}$ & $\begin{array}{l}3.50 \\
1\end{array}$ & $\begin{array}{l}2.71 \\
3\end{array}$ & & $\begin{array}{l}2.86 \\
8\end{array}$ & $\begin{array}{l}3.28 \\
6\end{array}$ & 3.287 & $\begin{array}{l}3.71 \\
5\end{array}$ & 2.764 & $\begin{array}{l}3.22 \\
1\end{array}$ \\
\hline R10 & $\begin{array}{l}.72 \\
6\end{array}$ & 3.501 & 3.694 & 2.86 & $\begin{array}{l}3.13 \\
2\end{array}$ & $\begin{array}{l}4.18 \\
5\end{array}$ & $\begin{array}{l}\mathbf{3 . 5 6} \\
3\end{array}$ & $\begin{array}{l}3.02 \\
7\end{array}$ & $\begin{array}{l}3.53 \\
9\end{array}$ & & 3.11 & $\mathbf{3 . 8 1 4}$ & $\begin{array}{l}\mathbf{3 . 5 1} \\
5\end{array}$ & 2.813 & $\begin{array}{l}3.21 \\
7\end{array}$ \\
\hline R11 & \begin{tabular}{|l|}
4.50 \\
1
\end{tabular} & 3.368 & $\begin{array}{l}.484 \\
\end{array}$ & $\begin{array}{l}2.55 \\
1\end{array}$ & $\begin{array}{l}2.71 \\
9\end{array}$ & $\begin{array}{l}4.02 \\
3\end{array}$ & $\begin{array}{l}3.53 \\
9\end{array}$ & $\begin{array}{l}2.94 \\
7\end{array}$ & $\begin{array}{l}3.50 \\
9\end{array}$ & $\begin{array}{l}2.41 \\
6\end{array}$ & & 3.706 & $\begin{array}{l}3.48 \\
7\end{array}$ & 2.948 & $\begin{array}{l}3.28 \\
6\end{array}$ \\
\hline R12 & \begin{tabular}{|l|}
4.37 \\
\end{tabular} & 3.326 & 3.392 & $\begin{array}{l}2.73 \\
8\end{array}$ & $\begin{array}{l}2.99 \\
5\end{array}$ & $\begin{array}{l}3.88 \\
5\end{array}$ & $\begin{array}{l}3.81 \\
8\end{array}$ & 3.4 & $\begin{array}{l}3.42 \\
2\end{array}$ & $\begin{array}{l}2.91 \\
2\end{array}$ & $\begin{array}{l}2.93 \\
9\end{array}$ & & $\begin{array}{l}3.73 \\
4\end{array}$ & 2.927 & $\begin{array}{l}3.48 \\
2\end{array}$ \\
\hline $\mathbf{R} 13$ & \begin{tabular}{|l|}
4.65 \\
8
\end{tabular} & 3.451 & 3.451 & $\begin{array}{l}2.73 \\
5\end{array}$ & $\begin{array}{l}3.07 \\
5\end{array}$ & $\begin{array}{l}4.01 \\
1\end{array}$ & $\begin{array}{l}3.20 \\
1\end{array}$ & $\begin{array}{l}2.62 \\
3\end{array}$ & $\begin{array}{l}3.19 \\
4\end{array}$ & $\begin{array}{l}2.72 \\
9\end{array}$ & 2.84 & 4.058 & & 3.117 & $\begin{array}{l}3.40 \\
2\end{array}$ \\
\hline R14 & \begin{tabular}{|l|}
4.63 \\
3
\end{tabular} & 3.506 & $\begin{array}{l}3.409 \\
\end{array}$ & $\begin{array}{l}\text { 3.08 } \\
7\end{array}$ & $\begin{array}{l}3.19 \\
3\end{array}$ & $\begin{array}{l}.07 \\
3\end{array}$ & $\begin{array}{l}3.72 \\
6\end{array}$ & $\begin{array}{l}3.03 \\
6\end{array}$ & $\begin{array}{l}3.21 \\
1\end{array}$ & $\begin{array}{l}2.95 \\
8\end{array}$ & $\begin{array}{l}2.96 \\
5\end{array}$ & 4.016 & $\begin{array}{l}3.55 \\
8\end{array}$ & & $\begin{array}{l}3.01 \\
7\end{array}$ \\
\hline R15 & \begin{tabular}{l|}
4.52 \\
6
\end{tabular} & 3.514 & $\begin{array}{l}3.588 \\
\end{array}$ & $\begin{array}{l}2.76 \\
7\end{array}$ & $\begin{array}{l}3.41 \\
6\end{array}$ & $\begin{array}{l}4.24 \\
5\end{array}$ & $\begin{array}{l}3.76 \\
1\end{array}$ & $\begin{array}{l}3.30 \\
4\end{array}$ & 3.56 & $\begin{array}{l}3.05 \\
5\end{array}$ & $\begin{array}{l}3.14 \\
6\end{array}$ & $\begin{array}{l}3.969 \\
\end{array}$ & $\begin{array}{l}3.55 \\
7\end{array}$ & 2.989 & \\
\hline
\end{tabular}

Table-1: Reputation Table

Direct Trust Simulation

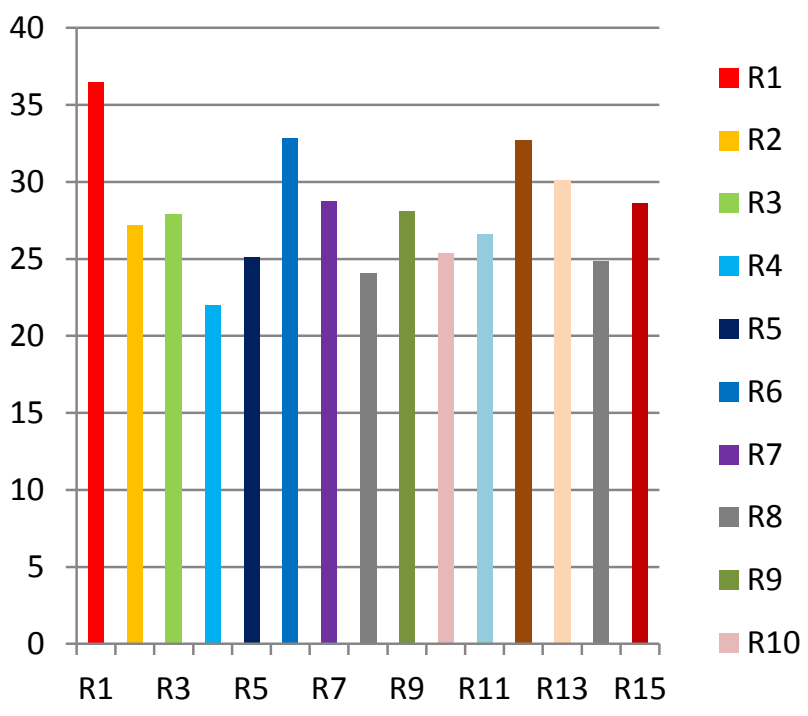

Figure-1: Histogram of Direct Trust
Total Trust simulation

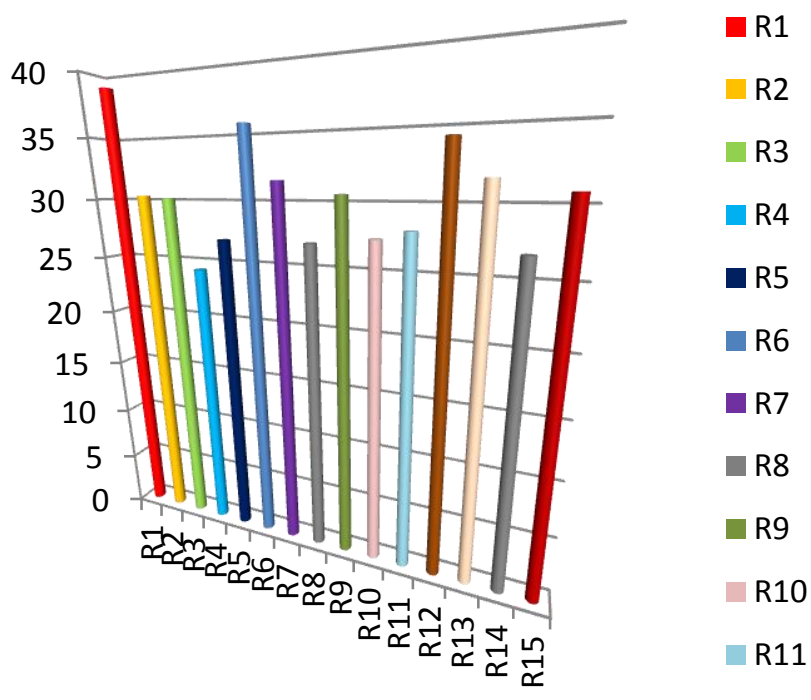

Figure-2: Histogram of Total Trust 


\begin{tabular}{|c|c|c|c|c|c|c|c|c|c|c|c|}
\hline \multicolumn{12}{|c|}{ Total Trust Table } \\
\hline \multirow{2}{*}{$\begin{array}{l}\text { Resourc } \\
\text { es }\end{array}$} & \multirow{2}{*}{$\begin{array}{c}\text { Direct } \\
\text { Trust( } \\
\text { DT) }\end{array}$} & \multicolumn{4}{|c|}{ Indirect Trust-1(ITSd) } & \multicolumn{4}{|c|}{ Indirect Trust-2(ITOd) } & \multirow{2}{*}{$\begin{array}{c}\text { Total } \\
\text { Trust(TT=DT+ } \\
\text { ITSd+ITOd) }\end{array}$} & \multirow{2}{*}{$\begin{array}{c}\operatorname{Max}(\mathbf{T} \\
\mathrm{T})\end{array}$} \\
\hline & & $\begin{array}{c}\text { Simila } \\
\text { rity }\end{array}$ & $\begin{array}{c}\text { Activit } \\
\mathbf{y}\end{array}$ & $\begin{array}{c}\text { Popula } \\
\text { rity }\end{array}$ & $\begin{array}{c}\text { Total(I } \\
\text { TSd) }\end{array}$ & $\begin{array}{c}\text { Simila } \\
\text { rity }\end{array}$ & $\begin{array}{c}\text { Activi } \\
\text { ty }\end{array}$ & $\begin{array}{c}\text { Popular } \\
\text { ity }\end{array}$ & $\begin{array}{l}\text { Total(I } \\
\text { TOd) }\end{array}$ & & \\
\hline $\mathbf{R 1}$ & $\begin{array}{c}36.4989 \\
13\end{array}$ & 1.967 & 0.0673 & $\begin{array}{c}0.1279 \\
2\end{array}$ & 2.16222 & 0 & $\begin{array}{c}0.071 \\
81\end{array}$ & 0.11469 & 0.1865 & 38.847633 & \multirow{15}{*}{$\begin{array}{c}38.847 \\
633 \text { i.e } \\
\text { The } \\
\text { Resour } \\
\text { ce R1 } \\
\text { is } \\
\text { Select }\end{array}$} \\
\hline $\mathbf{R 2}$ & 27.1885 & 2.51 & $\begin{array}{c}0.0744 \\
6 \\
\end{array}$ & $\begin{array}{c}0.1217 \\
2 \\
\end{array}$ & 2.70618 & 0.21 & $\begin{array}{c}0.117 \\
24 \\
\end{array}$ & 0.08192 & $\begin{array}{c}0.4091 \\
6 \\
\end{array}$ & 30.30384 & \\
\hline $\mathbf{R 3}$ & $\begin{array}{c}27.8998 \\
75\end{array}$ & 1.591 & $\begin{array}{c}\mathbf{0 . 1 8 4 7} \\
3\end{array}$ & 0.0864 & 1.86213 & 0.154 & 0.129 & 0.07627 & $\begin{array}{c}0.3592 \\
7\end{array}$ & 30.121275 & \\
\hline R4 & 21.986 & 1.832 & $\begin{array}{c}0.1035 \\
8 \\
\end{array}$ & 0.1284 & 2.06398 & 0.029 & $\begin{array}{c}0.116 \\
43 \\
\end{array}$ & 0.0548 & $\begin{array}{c}0.2002 \\
3 \\
\end{array}$ & 24.25021 & \\
\hline R5 & 25.075 & 1.188 & $\begin{array}{c}0.1336 \\
5\end{array}$ & $\begin{array}{c}0.1250 \\
6\end{array}$ & 1.44671 & 0.08 & $\begin{array}{c}0.138 \\
74\end{array}$ & 0.11808 & $\begin{array}{c}0.3368 \\
2\end{array}$ & 26.858553 & \\
\hline R6 & $\begin{array}{c}32.8341 \\
25 \\
\end{array}$ & 2.138 & $\begin{array}{c}0.1828 \\
2 \\
\end{array}$ & $\begin{array}{c}0.0935 \\
6 \\
\end{array}$ & 2.41438 & $\mathbf{0}$ & $\begin{array}{c}0.187 \\
83 \\
\end{array}$ & 0.17627 & 0.3641 & 35.612605 & \\
\hline R7 & 28.722 & 2.079 & $\begin{array}{c}0.1121 \\
7\end{array}$ & $\begin{array}{c}0.1317 \\
4 \\
\end{array}$ & 2.32291 & 0.11 & $\begin{array}{c}0.129 \\
41\end{array}$ & 0.2113 & $\begin{array}{c}0.4507 \\
1\end{array}$ & 31.49562 & \\
\hline $\mathbf{R 8}$ & $\begin{array}{c}24.0801 \\
8\end{array}$ & 1.965 & $\begin{array}{c}0.1412 \\
9\end{array}$ & $\begin{array}{c}0.1374 \\
7\end{array}$ & 2.24376 & 0.291 & $\begin{array}{c}0.109 \\
53\end{array}$ & 0.16667 & 0.5672 & 26.89114 & \\
\hline R9 & $\begin{array}{c}28.0858 \\
75\end{array}$ & 0.706 & $\begin{array}{c}0.1678 \\
1\end{array}$ & $\begin{array}{c}0.1551 \\
1\end{array}$ & 1.02892 & 0.43 & $\begin{array}{c}0.135 \\
59\end{array}$ & 0.8235 & $\begin{array}{c}1.3890 \\
9\end{array}$ & 30.503885 & \\
\hline $\mathbf{R} 10$ & $\begin{array}{c}25.3687 \\
5 \\
\end{array}$ & 1.565 & $\begin{array}{c}0.1609 \\
3 \\
\end{array}$ & $\begin{array}{c}0.0751 \\
7 \\
\end{array}$ & 1.8011 & $\mathbf{0}$ & $\begin{array}{c}0.110 \\
73 \\
\end{array}$ & 0.10588 & $\begin{array}{c}0.2166 \\
1 \\
\end{array}$ & 27.38646 & \\
\hline $\mathbf{R} 11$ & \begin{tabular}{c|}
26.5798 \\
75 \\
\end{tabular} & 0.997 & $\begin{array}{c}0.1191 \\
1 \\
\end{array}$ & $\begin{array}{c}0.0963 \\
5 \\
\end{array}$ & 1.21246 & $\mathbf{0}$ & $\begin{array}{c}0.096 \\
61 \\
\end{array}$ & 0.13387 & $\begin{array}{c}0.2304 \\
8 \\
\end{array}$ & 28.022815 & \\
\hline $\mathbf{R} 12$ & $\begin{array}{c}32.7146 \\
25 \\
\end{array}$ & 0.734 & $\begin{array}{c}0.1159 \\
3 \\
\end{array}$ & $\begin{array}{c}0.1371 \\
1 \\
\end{array}$ & 0.98704 & 0.144 & $\begin{array}{c}0.201 \\
13 \\
\end{array}$ & 0.14807 & 0.4932 & 34.194865 & \\
\hline R13 & $\begin{array}{c}30.1313 \\
75 \\
\end{array}$ & 0.534 & $\begin{array}{c}0.1064 \\
1 \\
\end{array}$ & $\begin{array}{c}0.2223 \\
4 \\
\end{array}$ & 0.86275 & 0.287 & $\begin{array}{c}0.128 \\
81 \\
\end{array}$ & 0.15456 & $\begin{array}{c}0.5703 \\
7 \\
\end{array}$ & 31.564495 & \\
\hline R14 & $\begin{array}{c}24.8267 \\
5 \\
\end{array}$ & 1.305 & $\begin{array}{c}0.1286 \\
4 \\
\end{array}$ & $\begin{array}{c}0.1773 \\
4 \\
\end{array}$ & 1.61098 & $\mathbf{0}$ & $\begin{array}{c}0.124 \\
29 \\
\end{array}$ & 0.18215 & $\begin{array}{c}0.3064 \\
4 \\
\end{array}$ & 26.74417 & \\
\hline R15 & $\begin{array}{c}28.6166 \\
3\end{array}$ & 1.389 & $\begin{array}{c}0.2011 \\
6\end{array}$ & $\begin{array}{c}0.1418 \\
7\end{array}$ & 1.73203 & 0 & $\begin{array}{c}0.202 \\
82\end{array}$ & 0.16876 & $\begin{array}{c}0.3715 \\
8\end{array}$ & 30.72024 & \\
\hline
\end{tabular}

Table-2: Total Trust Table

\section{Conclusion:}

In this paper we have presented a novel trust model which is comprehensive in the sense it considers and calculates individual reputation and on the basis of that direct trust and indirect trust of same domain and different domains calculated which provides more reliable and secure trust establishment. This model is unique in integrating various concepts that are important to the calculation of Total Trust values and the corresponding decisions to pick up or select the best resource in Grid environment. The underlying algorithm to calculate the trust is scalable and robust. We are going to work further on trust update algorithms that take into account the dynamics of trust.

\section{REFERENCES}

[1] http://searchdatacenter.techtarget.com/definition/gridcomputing.

[2] D. Gambetta. Can We Trust Trust? In [3], chapter 13, 1988.

[3] D. Gambetta (editor). Trust: Making and Breaking Cooperative Relations. Department of Sociology, University of Oxford, 1988. Available at http://www.sociology.ox.ac.uk/papers/trustbook.html .

[4] http://www.youtube.com/watch?v=7i5aU2pBcMY.

[5] L. Xiong, and L. Liu, "PeerTrust: Supporting ReputationBased Trust for Peer-to-Peer Electronic Communities," IEEE Transactions on Knowledge and Data Engineering, Vol. 16, No. 7, July 2004. 
[6] Y. Wang and J. Vassileva, "Trust and Reputation Model in Peer-to-Peer Networks," Proceedings of the Third International Conference on Peer-to-Peer Computing (P2P'03), 2003

[7] Selcuk, E. Uzun, and M. Pariente, "A Reputation-Based Trust Management System for P2P Networks," IEEE International Symposium on Cluster Computing and the Grid 2004

[8] Ayman Tajeddine Ayman Kayssi Ali Chehab Hassan Artail "A Comprehensive Reputation-Based Trust Model for Distributed Systems “ IEEE 2005.

[9] F.Azzedin,M.Maheswaran "Evolving and managing trust in grid computing system" IEEE CCECE,2002.

[10] Gui Xiaolin, Xie Bing "Study on behavior based trust model in grid security system" "Proceedings of the 2004 IEEE International Conference on Services Computing (SCC'04).

[11] Baolin Ma, Jizhou Sun, Ce Yu " Reputationbased Trust Model in Grid Security System “, Aug. 2006, Volume 3, No.8 (Serial No.21) Journal of Communication and Computer, ISSN1548-7709, USA.

[12] Beulah kurian, Gregor von laszewki "Reputation based grid resource selection"

[13] P.Srivaramangai , .R.Srinivasan " Reputation based trust model with elimination of unreliable feedbacks " International journal of information technology and knowledge management Vol2 , 2009. No 2,pp.455-459.

\section{AUTHOR'S PROFILE}

Mr. Sourav Gayen received his B.Sc (Math Hons) From B.C. College, West Bengal, Asansol, India and MCA(Master in Computer Application) from Asansol Engineering College., West Bengal, Asansol, India .He is currently pursuing MTech (CST) at Dr. B.C.Roy Engineering College.

He has worked in different technical positions for the last 4 years .Now he is working in Asansol Engineering College as a Technical Assistant since Aug'2010.

Mr. Avijit Bhowmick received his M.Tech from Bengal Engineering and Science University, Shibpur, West Bengal, India and MBA (Systems) from University of Kalyani, West Bengal, India. He is currently pursuing Ph.D (Computer Science \& Engineering).

He is working as Assistant Professor in the Dept. of CSE/IT at Dr.B.C.Roy Engineering College, Durgapur, West Bengal, India. His research interests includes distributed computing, network security, Grid computing, wireless network.

Mr.Bhowmick is a life member of Cryptology Research Society of India, Indian Society of Technical Education, IAENG and ACEEE.

Mr. Biswajit Upadhyay received his M.Tech from Dr. B.C.Roy Engineering College, Durgapur, ,West Bengal, India. And B.Tech from Asansol Engineering College, Asansol, West Bengal.

He is working as a Assistant Professor in the dept. of CSE/IT at Aryabhatta Institute of Engineering and Management, west Bengal, India. 